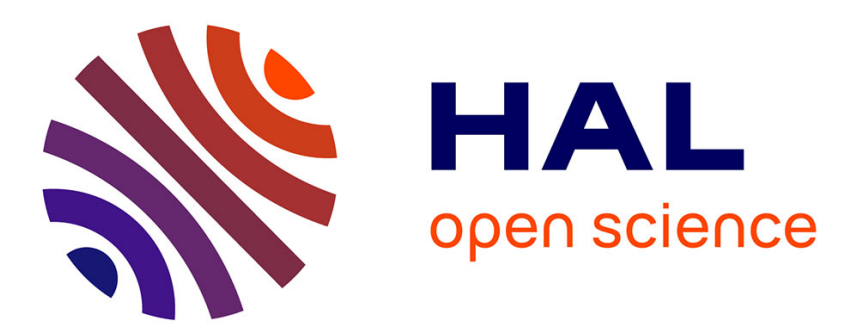

\title{
Effective properties of viscoelastic heterogeneous periodic media: An approximate solution accounting for the distribution of heterogeneities
}

\author{
H. Hoang-Duc, Guy Bonnet
}

\section{To cite this version:}

H. Hoang-Duc, Guy Bonnet. Effective properties of viscoelastic heterogeneous periodic media: An approximate solution accounting for the distribution of heterogeneities. Mechanics of Materials, 2013, 56 (/), pp.71-83. 10.1016/j.mechmat.2012.09.006 . hal-00764134

\section{HAL Id: hal-00764134 \\ https://hal.science/hal-00764134}

Submitted on 28 Jan 2016

HAL is a multi-disciplinary open access archive for the deposit and dissemination of scientific research documents, whether they are published or not. The documents may come from teaching and research institutions in France or abroad, or from public or private research centers.
L'archive ouverte pluridisciplinaire HAL, est destinée au dépôt et à la diffusion de documents scientifiques de niveau recherche, publiés ou non, émanant des établissements d'enseignement et de recherche français ou étrangers, des laboratoires publics ou privés. 


\title{
Effective properties of viscoelastic heterogeneous periodic media: An approximate solution accounting for the distribution of heterogeneities
}

\author{
H. Hoang-Duc, G. Bonnet* \\ Université Paris-Est, Laboratoire Modélisation et Simulation Multi Echelle, MSME UMR 8208 CNRS, 5 Bd Descartes, F-77454 Marne-la-Vallée Cedex 2, France
}

\section{A B S T R A C T}

This paper describes a simple approximate method for obtaining the viscoelastic properties of particle-matrix viscoelastic heterogeneous materials. This method accounts for the spatial distribution of heterogeneities. It rests on a formulation used for elastic media which allows, at the cost of evaluating parameters accounting for the distribution of heterogeneities, to compute the effective properties. Using the Laplace-Carson transform, the solution is shown as being a rational fraction of the Laplace variable, which allows for simple expressions of inverse Laplace transform. Some examples are shown for various viscoelastic behaviors in the case of viscoelastic media of different types, including a comparison with results coming from other methods.

Keywords:

Viscoelastic

Composites

Laplace transform

Fourier transform

Effective properties

\section{Introduction}

The prediction of the effective properties of heterogeneous media knowing the properties at the scale of the microstructure and their distribution has been the subject of numerous works since a few decades. More specifically, methods based on the Fourier transform have known an increasing interest, because these methods can be used on modern 3D images coming from tomography measurements. However, these methods have not been used in the case of viscoelastic constituents. Indeed, a classical way for obtaining viscoelastic solutions is to use the Laplace-Carson transform. However, results coming from fully numerical FFT solutions need a numerical inversion of Laplace transform, which would need the use of numerous applications of FFT computations at all values of Laplace variable. So, up to now, methods allowing to produce by simple ways the effective properties of viscoelastic media are restricted to simple methods like the Mori-Tanaka's model or the generalized self-consistent scheme for example. This paper presents a new method which allows the prediction of the

* Corresponding author. Tel.: +33160957220 .

E-mail address: Guy.Bonnet@univ-paris-est.fr (G. Bonnet). effective properties of inclusion-matrix heterogeneous media characterized by various distributions of heterogeneities. The method rests on an approximate FFT based solution producing the effective properties of elastic media (Iwakuma and Nemat-Nasser, 1983; Nemat-Nasser et al., 1993; Nemat-Nasser and Hori, 1993). The distribution of heterogeneities is taken into account by some synthetic structure parameters which are computed from the Fourier transform of the indicator function of the domain containing the heterogeneities. The work presented in this paper rests on an extension of this method to viscoelastic media.

The paper is organized as follows. The constitutive relation used for the individual viscoelastic constituents is presented in Section 2. Then, the approximated Fourier Transform solution is presented in the case of elasticity in Section 3. An important aspect is to evaluate the capacity of the method to compute accurately the properties of elastic periodic media. This is made by comparison with results coming from the application of the iterative FFT method in Section 4. The extension of the method to viscoelastic heterogeneous media is then treated in Section 5. The application to different kinds of viscoelastic behaviors, including a comparison with results of the literature (Lahellec and Suquet, 2007) coming from step by step time integration is finally shown in Section 6 . 


\section{Linear viscoelastic behavior}

2.1. Constitutive equations for an isotropic viscoelastic medium.

In the following, composite materials under study comprise at least one phase which is a non ageing viscoelastic material. The constitutive stress-strain relation of such a material is given classically (Christensen and Freund, 1984; Salençon, 2009) by a time integral relationship as:

$\boldsymbol{\sigma}(t)=\int_{0}^{t} \mathbb{R}(t-\tau): \frac{d \boldsymbol{\epsilon}(\tau)}{d \tau} d \tau=\mathbb{R}(: \circledast) \dot{\boldsymbol{\epsilon}}$

or reversely:

$\boldsymbol{\epsilon}(t)=\int_{0}^{t} \mathbb{J}(t-\tau): \frac{d \boldsymbol{\sigma}(\tau)}{d \tau} d \tau=\mathbb{J}(: \circledast) \dot{\boldsymbol{\sigma}}$

where $\mathbb{R}, \Downarrow$ are tensorial relaxation and creep functions. The convolution product of two functions $f$ and $g$, noted by " $f \circledast g$ ", is classically defined by:

$(f \circledast g)(x)=\int_{-\infty}^{+\infty} f(x-t) g(t) d t$

For a viscoelastic isotropic material, tensor $\mathbb{R}$ depends only on two scalar functions $R_{\kappa}(t)$ and $R_{\mu}(t)$ which are relaxation functions for compression and shear. The behavior of the material can then be expressed by using one of the following forms:

$\boldsymbol{\sigma}(t)=R_{\kappa}(t) \circledast \operatorname{tr} \boldsymbol{\epsilon}(t) \mathbf{1}+2 R_{\mu}(t) \circledast \mathbf{e}(t)$

$\boldsymbol{\epsilon}(t)=\frac{1}{9 R_{\kappa}(t)} \circledast \operatorname{tr} \boldsymbol{\sigma}(t) \mathbf{1}+\frac{1}{2 R_{\mu}(t)} \circledast \mathbf{S}(t)$

where $\mathbf{s}$ and $\mathbf{e}$ are deviators of stress and strain tensors.

\subsection{Laplace-Carson transform}

The Laplace-Carson transform $f^{*}(p)$ of a real function $f(t), t \geqslant 0$ is obtained from its Laplace transform $\tilde{f}(s)$ by:

$f^{*}(s)=s \tilde{f}(s)=s \int_{0}^{\infty} e^{-s t} f(t) d t$

Effecting the Laplace Carson transform of the first expression in (4) leads to:

$\boldsymbol{\sigma}^{*}(s)=R_{\kappa}^{*}(s) \operatorname{tr} \boldsymbol{\epsilon}(s) \mathbf{1}+2 R_{\mu}^{*}(s) \mathbf{e}^{*}(s)$

which provides also:

$\epsilon^{*}(s)=\frac{1}{9 R_{\kappa}^{*}(s)} \operatorname{tr} \boldsymbol{\sigma}^{*}(s) \mathbf{1}+\frac{1}{2 R_{\mu}^{*}(s)} \mathbf{s}^{*}(s)$

where $s$ is the variable in Laplace-Carson space.

As it is well-known, these expressions show that for any fixed value of $s$, the stress-strain relation in Laplace-Carson space is formally equivalent to the elasticity constitutive equation of an isotropic elastic material. This is named as "pseudo-elastic" behavior. This is the base of many solutions obtained for viscoelastic problems. However, having obtained the pseudo-elastic solution, the recovery of the viscoelastic solution needs to perform its inverse Laplace transform. Even if numerical inversion methods exist, the pseudo-elastic solution presents an interest essentially in the case where the inverse Laplace transform can be obtained by simple ways. This is the case when the pseudoelastic solution has the form of a rational fraction, as shown thereafter.

2.3. Inverse Laplace transform of the pseudo-elastic solution having the form of a rational fraction

Solving the viscoelastic problem is necessary to inverse the solution obtained in the Laplace space. If this solution $F(s)$ is a rational fraction, the numerator $P(s)$ and the denominator $Q(s)$ are both polynomial functions. $Q(s)$ is assumed to possess $n$ distinct zero $\alpha_{k}, k=1,2,3, \ldots, n$ and to be such that the degree of $P(s)$ is less than the degree of $Q(s)$.

Then:

$L^{-1}\{F(s)\}=L^{-1}\left\{\frac{P(s)}{Q(s)}\right\}=\sum_{k=1}^{n} \frac{P\left(\alpha_{k}\right)}{Q^{\prime}\left(\alpha_{k}\right)} e^{\alpha_{k} t}$

In the case where $Q(s)=0$ has a multiple root of order $m$, while the other roots, $\beta_{1}, \beta_{2}, \ldots, \beta_{n}$ are simple, $F$ can be developed as:

$\frac{P(s)}{Q(s)}=\sum_{k=1}^{m} \frac{A_{k}}{(s-\alpha)^{m-k+1}}+\sum_{k=1}^{n} \frac{B_{k}}{\left(s-\beta_{k}\right)}$

Then, the inverse of the Laplace transform is given by:

$L^{-1}\left\{\frac{P(s)}{Q(s)}\right\}=e^{\alpha t}\left\{\sum_{r=1}^{m} \frac{A_{r} t^{m-r}}{(m-r) !}\right\}+\sum_{r=1}^{n} B_{r} e^{\beta_{r} t}$

where:

$A_{k}=\lim _{s \rightarrow \alpha} \frac{1}{(k-1) !} \frac{d^{k-1}}{d s^{k-1}}\left\{(s-\alpha)^{m} f(s)\right\}$

\section{Overall elastic properties of periodic elastic composites}

\subsection{Basic equation of the problem}

Let us consider an elastic composite material made of periodic cells as in Fig. 1. The periodic medium is obtained by a periodic translation of the cell along different directions of the space.

Let us denote by $2 a_{i}(i=1,2,3)$, the dimension of a basic unit parallelepipedic cell along direction $x_{i}$. Then the dis-

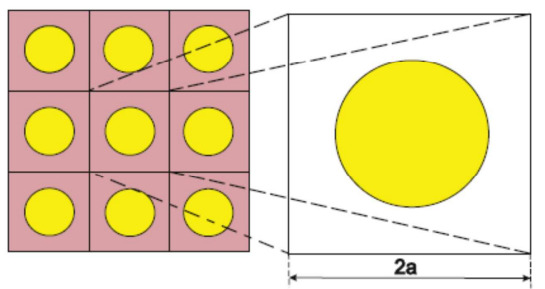

Fig. 1. Basic cell of a heterogeneous periodic medium. 
placement field $\mathbf{u}=\mathbf{u}(\mathbf{x})$, strain field $\boldsymbol{\epsilon}=\boldsymbol{\epsilon}(\mathbf{x})$ and stress field $\boldsymbol{\sigma}=\boldsymbol{\sigma}(\mathbf{x})$ comply with the following relationships:

$$
\left\{\begin{array}{l}
\boldsymbol{\epsilon}(\mathbf{x})=\frac{1}{2}\left\{\boldsymbol{\nabla} \otimes \mathbf{u}(\mathbf{x})+(\boldsymbol{\epsilon} \otimes \mathbf{u}(\mathbf{x}))^{t}\right\} \\
\nabla . \boldsymbol{\sigma}(\mathbf{x})=\mathbf{0} \\
\boldsymbol{\sigma}(\mathbf{x})=\mathbb{C}(\mathbf{x}): \boldsymbol{\epsilon}(\mathbf{x}) \\
\mathbf{u}(\mathbf{x})=\mathbf{E} \cdot \mathbf{x}+\mathbf{u}_{\text {per }}(\mathbf{x})
\end{array}\right.
$$

where the displacement field $\mathbf{u}_{\text {per }}$ is periodic and $\mathbb{C}(\mathbf{x})$ is the elasticity tensor which is also satisfying a periodicity condition:

$$
\left\{\begin{array}{l}
\mathbb{C}(\mathbf{x})=\mathbb{C}(\mathbf{x}+\mathbf{d}) \\
\mathbf{d}=\sum_{i=1}^{3} 2 n_{i} a_{i} \mathbf{e}_{i}
\end{array}\right.
$$

where $n_{i}$ is an arbitrary integer. $\mathbf{u}^{\text {per }}$ is a periodic displacement field and hence strain and stress fields are also periodic:

$$
\left\{\begin{array}{l}
\boldsymbol{\sigma}(\mathbf{x})=\boldsymbol{\sigma}(\mathbf{x}+\mathbf{d}) \\
\boldsymbol{\epsilon}(\mathbf{x})=\boldsymbol{\epsilon}(\mathbf{x}+\mathbf{d})=\mathbf{E}+\boldsymbol{\epsilon}_{p e r}
\end{array}\right.
$$

\subsection{Strain and stress fields in Fourier space}

Because of the periodicity of the medium, the solution can be developed into Fourier series, as proposed in references (Iwakuma and Nemat-Nasser, 1983; Michel et al., 1999) or (Moulinec and Suquet, 2003). This series is the discrete form of the Fourier transform of a periodic function.

Let us consider a periodic function $f(\mathbf{x})$ defined on the cell $V$ defined by:

$V=\left\{\mathbf{x},-a_{j} \leqslant x_{j} \leqslant a_{j}(j=1,2,3)\right\}$

with the condition of periodicity: $f(\mathbf{x})=f(\mathbf{x}+\mathbf{d})$

This function can be expanded into Fourier series as follows:

$f(\mathbf{x})=\sum_{\xi} \hat{f}(\xi) e^{i \xi x}, \quad i=\sqrt{-1}$

with:

$\hat{f}(\boldsymbol{\xi})=\frac{1}{V} \int_{V} f(\mathbf{x}) e^{-i \xi \mathbf{x}} d V, \quad \xi_{j}=\frac{\pi n_{j}}{a_{j}}, \quad($ no sum on $\mathrm{j})$

Let us consider the periodic part $\mathbf{u}_{\text {per }}$ of the displacement field $\mathbf{u}$, whose constant part is assumed null:

$\mathbf{u}_{\text {per }}(\mathbf{x})=\sum_{\xi}^{\prime} \widehat{\mathbf{u}_{p e r}}(\boldsymbol{\xi}) e^{i \xi \mathbf{x}}$

where a prime on $\sum$ indicates that the null wave vector is excluded from the summation. Each Fourier component is given by:

$\widehat{\mathbf{u}_{p e r}}(\boldsymbol{\xi})=\frac{1}{V} \int_{V} \mathbf{u}_{\text {per }}(\mathbf{x}) e^{-i \xi \mathbf{x}} d \mathbf{x}$

Differentiating this periodic field produces the "periodic strain field" $\epsilon_{\text {per }}$ whose Fourier expansion is:

$\boldsymbol{\epsilon}_{\text {per }}(\mathbf{x})=\sum_{\xi}^{\prime} \widehat{\boldsymbol{\epsilon}}_{p e r}(\boldsymbol{\xi}) e^{i \xi \mathbf{x}}$
Finally, the relation between these "periodic parts" simultaneously with the constitutive elasticity in Fourier space are:

$\widehat{\boldsymbol{\epsilon}}_{\text {per }}(\boldsymbol{\xi})=\frac{i}{2}\left\{\boldsymbol{\xi} \otimes \widehat{\mathbf{u}_{\text {per }}}(\boldsymbol{\xi})+\widehat{\mathbf{u}_{\text {per }}}(\boldsymbol{\xi}) \otimes \boldsymbol{\xi}\right\}$

$\widehat{\boldsymbol{\sigma}}(\boldsymbol{\xi})=i \sum \widehat{\mathbb{C}}(\boldsymbol{\xi}-\boldsymbol{\zeta}):\{\boldsymbol{\zeta} \otimes \widehat{\mathbf{u}}(\boldsymbol{\zeta})\}$

3.3. Obtertion of the effective properties

The effective properties come from the solution of the set of Eq. (13). This solution is sought by using a constant "reference elasticity tensor" $\mathbb{C}^{0}$. The expression of the local stress field is transformed as:

$\mathbb{C}(\mathbf{x}):\left(\mathbf{E}+\boldsymbol{\epsilon}_{\text {per }}(\mathbf{x})\right)=\mathbb{C}^{0}:\left(\mathbf{E}+\boldsymbol{\epsilon}_{\text {per }}(\mathbf{x})-\boldsymbol{\epsilon}^{L}(\mathbf{x})\right)$

where the "free strain" $\epsilon^{L}$ has been introduced. Inserting this relation into the equilibrium equation transforms the elasticity problem for a heterogeneous medium into an elasticity problem for a homogeneous medium and free strains. The interest of this expression is to produce the value of the "periodic strain field" induced by the free strain under the form:

$\widehat{\boldsymbol{\epsilon}}_{\text {per }}(\boldsymbol{\xi})=\widehat{\mathbb{S}}(\boldsymbol{\xi}): \widehat{\boldsymbol{\epsilon}}^{L}(\boldsymbol{\xi})$

where tensor $\widehat{\mathbb{S}}(\boldsymbol{\xi})$ is given by:

$\widehat{\mathbb{S}}(\xi)=\widehat{\Gamma}(\xi): \mathbb{C}^{0}$

as a function of the more commonly used "strain Green's tensor" $\widehat{\boldsymbol{\Gamma}}(\boldsymbol{\xi})$ whose expression is known explicitly as a function of the reference elasticity tensor and of the wave vectors. As an example, for an isotropic material this tensor is transversely isotropic and can be expressed in the Walpole's basis (Walpole, 1981 and Walpole et al., 1966) as shown in Appendix B, by:

$\widehat{\Gamma}(\xi)=\frac{1}{\lambda_{0}+2 \mu_{0}} \mathbb{E}_{2}(\xi)+\frac{1}{2 \mu_{0}} \mathbb{E}_{4}(\xi)$

where $\mathbb{E}_{2}$ and $\mathbb{E}_{4}$ are tensors of the Walpole's basis.

Introducing this expression of the periodic strain field into (23) produces:

$\delta \mathbb{C}:\left(\mathbf{E}+\sum_{\xi}^{\prime} \widehat{\mathbb{S}}(\boldsymbol{\xi}): \psi\right)=\mathbb{C}^{0}: \epsilon^{L}(\mathbf{x})$

with:

$\left\{\begin{array}{l}\psi=\frac{1}{V} \int_{V} \epsilon^{L}\left(\mathbf{x}^{\prime}\right) e^{i \xi\left(\mathbf{x}-\mathbf{x}^{\prime}\right)} d V_{\mathbf{x}^{\prime}} \\ \delta \mathbb{C}=\mathbb{C}^{0}-\mathbb{C}(\mathbf{x})\end{array}\right.$

The problem is now to obtain the free field by solving this "integral equation" (due to the integral appearing in $\psi)$. One usual way is to solve this integral equation by successive iterations (Michel et al., 1999; Moulinec and Suquet, 2003; Zeman et al., 2010). However, in order to provide possible solutions in the case of viscoelasticity, a simple approximate solution for the effective properties will be obtained in the following section for the volume average of the "free strain" on the domain of the inclusions. 


\subsection{An approximation of the solution of the integral equation}

Let us consider a basic cell $D$ which consists of a matrix of linear elasticity tensor $\mathbb{C}^{M}$ containing inclusions $\Omega$ of elasticity tensor $\mathbb{C}^{l}$. The approximation introduced in reference (Iwakuma and Nemat-Nasser, 1983) is to replace the eigenstrain field $\epsilon^{L}$ by its volume average under the integral appearing in the volume average of the previous relation, when using a reference medium equal to the elasticity tensor of the matrix. This leads to:

$\mathbb{C}^{M}:\left\langle\boldsymbol{\epsilon}^{L}(\mathbf{x})\right\rangle=\delta \mathbb{C}:\left(\mathbf{E}+\sum_{\xi}^{\prime} P(\boldsymbol{\xi}) \widehat{\mathbb{S}}(\boldsymbol{\xi}):\left\langle\boldsymbol{\epsilon}^{L}(\mathbf{x})\right\rangle\right)$

where $\delta \mathbb{C}=\mathbb{C}^{M}-\mathbb{C}^{I}$ and the brackets denote the volume average over the cell. Function $P(\xi)$ is defined by:

$P(\xi)=f\left\{\frac{1}{V_{\Omega}} I(\xi)\right\}\left\{\frac{1}{V_{\Omega}} I(-\xi)\right\}$

which uses the volume fraction of inclusions $f$ and a shape function $I(\xi)$ which depends explicitly on the distribution of inclusions within the periodic cell, being defined by:

$I(\xi)=\int_{\Omega} e^{i \xi \mathbf{x}} d \mathbf{x}$

on the volume $\Omega$ of the inclusions.

The relation between the macroscopic strain $\mathbf{E}$ and the volume average of the free strain becomes:

$[\mathbb{A}-\mathbb{Q}]:\left\langle\boldsymbol{\epsilon}^{L}(\mathbf{x})\right\rangle=\mathbf{E}$

where:

$$
\left\{\begin{array}{l}
\mathbb{A}=\delta \mathbb{C}^{-1}: \mathbb{C}^{M} \\
\mathbb{Q}=\sum_{\xi}^{\prime} P(\xi) \widehat{\mathbb{S}}(\xi)
\end{array}\right.
$$

Now, taking the volume average of Eq. (24) produces the volume average of the stress field, which is related to the macroscopic strain by the effective elasticity tensor, leading to:

$$
\mathbb{C}^{e f f}: \mathbf{E}=\mathbb{C}^{M}: \mathbf{E}-f \mathbb{C}^{M}:\left\langle\boldsymbol{\epsilon}^{L}(\mathbf{x})\right\rangle
$$

From Eqs. (32) and (34) used for any value of the macroscopic strain, the effective modulus is solution of a linear equation:

$f \mathbb{C}^{M}=\left(\mathbb{C}^{M}-\mathbb{C}^{e f f}\right):(\mathbb{A}-\mathbb{Q})$

By using Eq. (33), it becomes:

$f \mathbb{\square}=\left(\mathbb{C}^{M}-\mathbb{C}^{e f f}\right):\left(\delta \mathbb{C}^{-1}-\sum_{\xi}^{\prime} P(\xi) \widehat{\Gamma}(\xi)\right)$

This last expression produces an explicit relation allowing the computation of the effective elasticity tensor for any distribution of heterogeneities (through function $P(\xi)$ ). Obviously, the accuracy of the approximate solution obtained in this way must be compared with the full solution of the integral equation in order to know in which case such a solution can be used. This is the objective of the following section.

\section{Two examples of elastic composites and comparison} with a complete solution

\subsection{A cubic array of spheres}

For a first illustration, we consider a periodic composite having cubic symmetry. Its basic cell is a cube of size $\Lambda$ containing a centered spherical inclusion of radius $R$. Let the material of the cell be linearly isotropic elastic with the elasticity tensor $\mathbb{C}$. The solution of Eq. (36) will be made clearer by using matrix expressions of fourth-rank tensors.

We consider the fourth-rank elasticity tensor $\mathbb{L}$ of components $L_{i j k l}$ which possesses the major symmetry and the minor symmetry related to couples $(i, j)$ and $(k, l)$. The tensorial relationship between second order tensors $\mathbf{a}$ and $\mathbf{b}$ using tensor $\mathbb{L}$ is given by:

$\mathbf{b}=\mathbb{L}: \mathbf{a}$

This can be translated into the matrix form:

$$
\left[B_{\alpha}\right]=\left[L_{\alpha \beta}\right]\left[A_{\beta}\right]
$$

where:

$[B]=\left[\begin{array}{r}b_{11} \\ b_{22} \\ b_{33} \\ \sqrt{2} \cdot b_{23} \\ \sqrt{2} \cdot b_{31} \\ \sqrt{2} \cdot b_{12}\end{array}\right] \quad[A]=\left[\begin{array}{r}a_{11} \\ a_{22} \\ a_{33} \\ \sqrt{2} \cdot a_{23} \\ \sqrt{2} \cdot a_{31} \\ \sqrt{2} \cdot a_{12}\end{array}\right]$

$[L]=\left[\begin{array}{ll}{\left[L^{11}\right]} & {\left[L^{12}\right]} \\ {\left[L^{12}\right]} & {\left[L^{22}\right]}\end{array}\right]$

with:

$$
\begin{aligned}
& {\left[L^{11}\right]=\left[\begin{array}{lll}
L_{1111} & L_{1122} & L_{1133} \\
L_{2211} & L_{2222} & L_{2233} \\
L_{3311} & L_{3322} & L_{3333}
\end{array}\right]} \\
& {\left[L^{12}\right]=\sqrt{2}\left[\begin{array}{lll}
L_{1123} & L_{1131} & L_{1112} \\
L_{2223} & L_{2231} & L_{2212} \\
L_{3323} & L_{3331} & L_{3312}
\end{array}\right]}
\end{aligned}
$$

$\left[L^{22}\right]=2\left[\begin{array}{lll}L_{2323} & L_{2331} & L_{2312} \\ L_{3123} & L_{3131} & L_{3112} \\ L_{1223} & L_{1231} & L_{1212}\end{array}\right]$

Eq. (36) can be rewritten as follows:

$f \square=\left(\mathbb{C}^{M}-\mathbb{C}^{\text {eff }}\right):\left(\left(\mathbb{C}^{M}-\mathbb{C}^{I^{-1}}-\mathbb{S}_{1}\right)\right.$

where

$\mathbb{S}_{1}=\frac{1}{\lambda_{M}+2 \mu_{M}} \mathbb{U}+\frac{1}{2 \mu_{M}} \mathbb{V}$ 
with:

$$
\left\{\begin{array}{l}
\mathbb{U}=\sum_{\xi}^{\prime} P(\xi) \mathbb{E}_{2}(\xi) \\
\mathbb{V}=\sum_{\xi}^{\prime} P(\xi) \mathbb{E}_{4}(\xi)
\end{array}\right.
$$

The matrix expression of the effective elasticity tensor is finally given by:

$$
\left[\mathbf{C}^{\text {eff }}\right]=\left[\mathbf{C}^{M}\right]-f[\mathbf{D}]^{-1}
$$

with:

$$
[\mathbf{D}]=\left[\delta \mathbf{C}^{-1}-\frac{1}{\lambda_{M}+2 \mu_{M}}[\mathbf{U}]-\frac{1}{2 \mu_{M}}[\mathbf{V}]\right.
$$

Tensors $\mathbb{U}$ and $\mathbb{V}$ are obtained from tensors $\mathbb{E}_{2}$ and $\mathbb{E}_{4}$ which themselves can be expressed by using two tensors $\mathbb{G}^{(1)}$ and $\mathbb{G}^{(2)}$. This expression is given in matrix form by:

$$
\left\{\begin{array}{l}
{\left[\mathbf{E}_{2}\right]=\left[\mathbf{G}^{(2)}\right]} \\
{\left[\mathbf{E}_{4}\right]=\left[\mathbf{G}^{(1)}\right]-2\left[\mathbf{G}^{(2)}\right]}
\end{array}\right.
$$

The components of matrix $\left[\mathbf{G}^{(1)}\right],\left[\mathbf{G}^{(2)}\right]$ are given in (C).

These components are functions of nine scalar functions of the wave vectors $h_{k}(\xi), k=1 \ldots 9$. Finally, matrices $[\mathbf{U}]$ and $[\mathbf{V}]$ are functions of nine constants $S_{k}, k=1 \ldots 9$, with $S_{k}=\sum P(\xi) h_{k}(\xi)$

For the chosen cubic symmetry, it can be noticed that matrices $[\delta \mathbf{C}]^{-1},[\mathbf{U}],[\mathbf{V}]$ can be diagonalized by using the same eigenvectors. It allows to compute the inverse of matrix $[\mathbf{D}]$ in the form:

$[\mathbf{D}]^{-1}=[\mathbf{Q}]\left(\left[\mathbf{D}_{a}\right]-\frac{1}{\lambda_{M}+2 \mu_{M}}\left[\mathbf{D}_{u}\right]-\frac{1}{2 \mu_{M}}\left[\mathbf{D}_{v}\right]\right)[\mathbf{Q}]^{-1}$

where: $\left[\mathbf{D}_{a}\right],\left[\mathbf{D}_{u}\right],\left[\mathbf{D}_{v}\right]$ are the diagonal matrices containing eigenvalues $a_{k}, u_{k}, v_{k}$ of matrix $[\delta \mathbf{C}]^{-1},[\mathbf{U}],[\mathbf{V}]$.

$[\mathbf{Q}]$ is the matrix of base change given by:

$$
[\mathbf{Q}]=\left[\begin{array}{cccccc}
1 & -1 & -1 & 0 & 0 & 0 \\
1 & 0 & 1 & 0 & 0 & 0 \\
1 & 1 & 0 & 0 & 0 & 0 \\
0 & 0 & 0 & 1 & 0 & 0 \\
0 & 0 & 0 & 0 & 1 & 0 \\
0 & 0 & 0 & 0 & 0 & 1
\end{array}\right]
$$

Finally, the matrix associated with the overall elasticity tensor is given by:

$$
\left[\mathbf{C}^{\text {eff }}\right]=\left[\mathbf{C}^{M}\right]-f r_{k}\left[\mathbf{H}_{(k)}\right]
$$

with:

$$
\begin{aligned}
& {\left[\mathbf{H}_{(k)}\right]=[\mathbf{Q}]\left[\mathbf{F}_{(k)}\right][\mathbf{Q}]^{-1}} \\
& r_{k}=\frac{1}{a_{k}-\frac{u_{k}}{\lambda_{M}+2 \mu_{M}}-\frac{v_{k}}{2 \mu_{M}}} F_{(k) i j}=\delta_{i k} \delta_{j k}
\end{aligned}
$$

where the matrices $\left[\mathbf{H}_{(k)}\right]$ depend only on the geometry of the inclusions and of the cell.
It can be noticed that this expression takes the form of a rational fraction of the elastic modulus of the components, which will be the main property used for producing the extension to the effective properties of viscoelastic composites in the following section.

The shape function which must be introduced into Eq. (31) for the particular geometry of the simple cubic array of spheres is given by Nemat-Nasser et al. (1982):

$\frac{1}{V_{\Omega}} I(\xi)=\frac{3(\sin \eta-\eta \cos \eta)}{\eta 3} \quad(\eta \neq 0)$

with

$\left\{\begin{array}{l}\eta=2 \pi\left(\frac{3 f}{4 \pi}\right)^{1 / 3}\left(n_{k} n_{k}\right)^{1 / 2} \\ \bar{\xi}_{j}=\frac{n_{j}}{\left(n_{k} n_{k}\right)^{1 / 2}}\end{array}\right.$

Another simplification comes from the symmetry of the cell, because the independent values of coefficients $S_{k}$ which appear in the computation of matrices $[\mathbf{U}]$ and $[\mathbf{V}]$ are only three, due to:

$\left\{\begin{array}{l}S_{1}=S_{2}=S_{3} \\ S_{4}=S_{5}=S_{6} \\ S_{7}=S_{8}=S_{9}\end{array}\right.$

The homogenized material has a cubic symmetry. Consequently, the effective elasticity tensor is defined by three independent coefficients:

- The effective incompressibility modulus $\kappa_{\text {eff }}$ defined by:

$$
\kappa_{\text {eff }}=\frac{C_{1111}^{\text {eff }}+2 C_{1122}^{\text {eff }}}{3}
$$

- The first effective shear module $\mu_{\text {eff }}$ defined by:

$$
\mu_{\text {eff }}=C_{2323}^{\text {eff }}
$$

- The second effective shear module $\mu_{\text {eff }}^{*}$ given by:

$$
\left(\Sigma_{11}-\Sigma_{22}\right)=2 \mu_{\text {eff }}^{*}\left(E_{11}-E_{22}\right)
$$

or

$$
\mu_{\text {eff }}^{*}=\frac{C_{1111}^{\text {eff }}-C_{1122}^{\text {eff }}}{2}
$$

Then, an application is performed on a specific composite whose elastic properties of phases are given in Table 1.

Figs. 2 and 3 show the values of the first effective shear modulus and of the incompressibility modulus as functions of the concentration of the spherical inclusions, compared with the full FFT solution of the integral equation and to the bounds of Voigt and Reuss, showing that these vales are coherent with first-order bounds and that the

Table 1

Elastic properties of the composite.

\begin{tabular}{lll}
\hline Elastic modulus $(\mathrm{GPa})$ & Matrix & Inclusion \\
\hline Incompressibility modulus $\kappa$ & 30 & 100 \\
Shear modulus $\mu$ & 20 & 50 \\
\hline
\end{tabular}




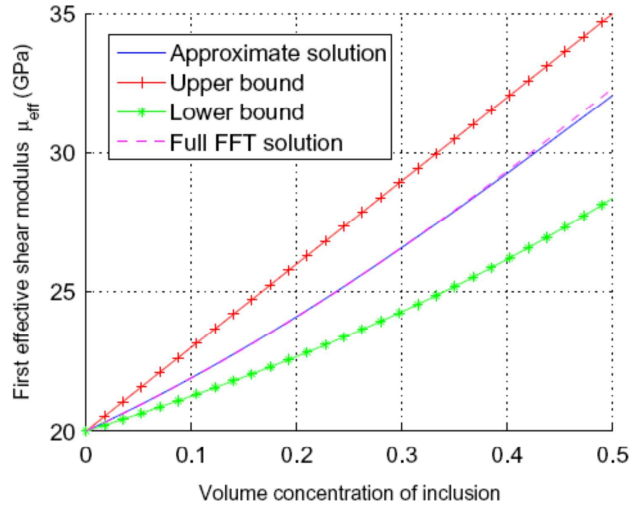

Fig. 2. Estimate of the first effective shear modulus for a simple cubic array of spheres. Comparison with the full FFT solution and with the bounds of Voigt and Reuss.

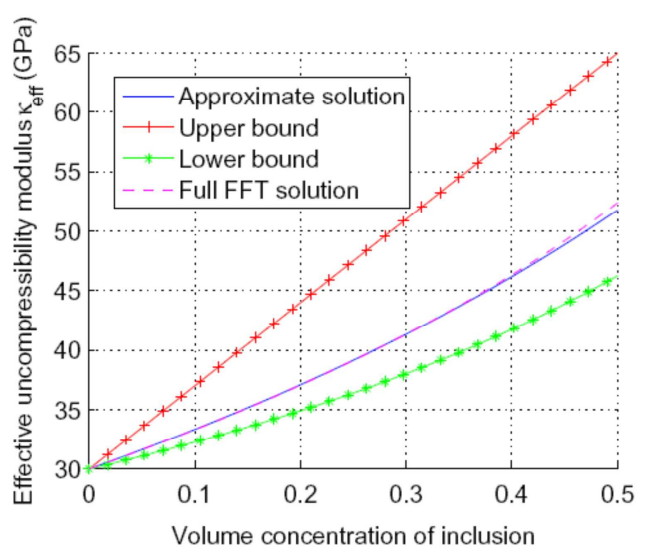

Fig. 3. Estimate of the incompressibility modulus for a simple cubic array of spheres. Comparison with the full FFT solution and with bounds of Voigt and Reuss.

approximate solution provides a very good approximation on the full range of concentrations.

The calculation of the effective properties depends on the computation of coefficients $S_{k}$ which come from infinite summations on all wave numbers. Finally, the result depends on the quantity of wave numbers which is used in the summation. Figs. 4 and 5 show the values of effective modulus obtained for different choices of the number of wave vectors. These figures show that a wave number in the formula (18) with $n_{j} \in[-64: 63]$ for each direction, giving a total number of waves vectors: $128 \times 128 \times 128$, ensures the convergence of the sums appearing in the expression of the overall modulus.

It is worthwhile noticing that it was found that the quantity of wave-numbers for obtaining the convergence is higher than the one which produces convergence when using the complete FFT solution.

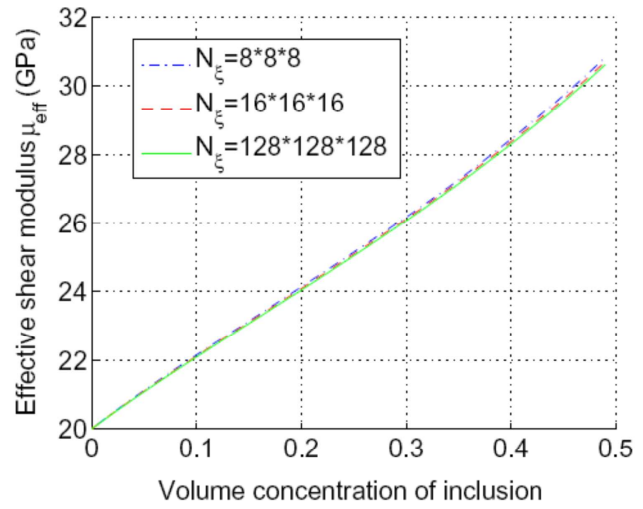

Fig. 4. Study of the convergence for different numbers of wave vectors when computing the first shear modulus.

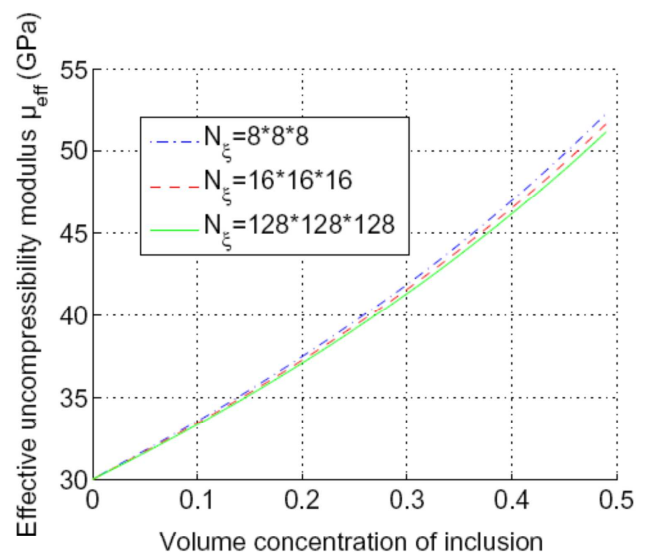

Fig. 5. Study of the convergence for different numbers of wave vectors when computing the incompressiblility modulus.

\subsection{A squared array of fibers}

The second example which is considered is a composite containing a squared array of fibers, both matrix and fibers being incompressible. The comparison is made with the results of complete FFT iterative scheme, but using the exact shape functions, as performed in Bonnet (2007). The shear modulus of the matrix is $\mu_{\mathrm{M}}=70 \mathrm{GPa}$ and the one of the inclusion is $\mu_{l}=5 \mathrm{GPa}$.

The shape functions related to the squared array of fibers to be introduced into Eq. (31) are given by Nemat-Nasser et al. (1982):

$I_{\alpha}(\xi)=\frac{2 \cdot S_{\alpha} J_{1}(\eta)}{\eta}$

where $J_{1}$ is the Bessel function of first order. $S_{\alpha}$ is the area of inclusion, $\eta$ is given by: 
$\eta=R\left(\xi_{1}^{2}+\xi_{2}^{2}\right)^{1 / 2}$

where, $R$ is the radius of the fiber

The Green's tensor is given in the incompressible case by:

$\widehat{\boldsymbol{\Gamma}}(\boldsymbol{\xi})=\frac{\mathbb{E}_{4}}{2 \mu}$

The non null components of the symmetric tensor $\mathbb{E}_{4}$ are determined by:

$$
\begin{array}{ll}
E_{1111}=4\left(\xi_{1}^{2}-\xi_{1}^{4}\right) & E_{2222}=4\left(\xi_{2}^{2}-\xi_{2}^{4}\right) \\
E_{1122}=-4 \xi_{1}^{2} \xi_{2}^{2} & E_{1112}=2 \xi_{1} \xi_{2}\left(1-2 \xi_{1}^{2}\right) \\
E_{3131}=\xi_{1}^{2} & E_{1212}=\xi_{1}^{2}+\xi_{2}^{2}-4 \xi_{1}^{2} \xi_{2}^{2} \\
E_{2323}=\xi_{2}^{2} & E_{2212}=2 \xi_{1} \xi_{2}\left(1-2 \xi_{2}^{2}\right)
\end{array}
$$

Fig. 6 shows the comparison between the shear modulus obtained from the approximate solution and from the complete FFT solution. Results show that the approximate solution is close to the full FFT solution up to concentrations which are close to 0.5 . From a more general point of view, the precision of the approximate method depends on the contrast $\alpha=\frac{\mu_{I}}{\mu_{M}}$ between inclusion and matrix. Fig. 7 shows the error coming from the approximate solution as a function of the contrast and shows that the error increases with the contrast, but remains small for all contrasts at concentrations inferior to 0.3 . The results presented here correspond to contrasts inferior to 1 , but similar results are obtained for contrasts superior to 1 . Another important parameter is the number of wave vectors which are used in the computation. The number of wave-vectors used for the approximate solution is $256 \times 256$, while the one used for the FFT solution is $64 \times 64$. Generally, it was found that the number of wave-vectors for reaching convergence is higher for the approximate solution than for the full FFT solution.

The result show that the quality of the approximate solution depends not only of volume concentration but also on the contrast of shear moduli. However, for concentrations lower than 0.3 , the approximate solution is accurate for any case.

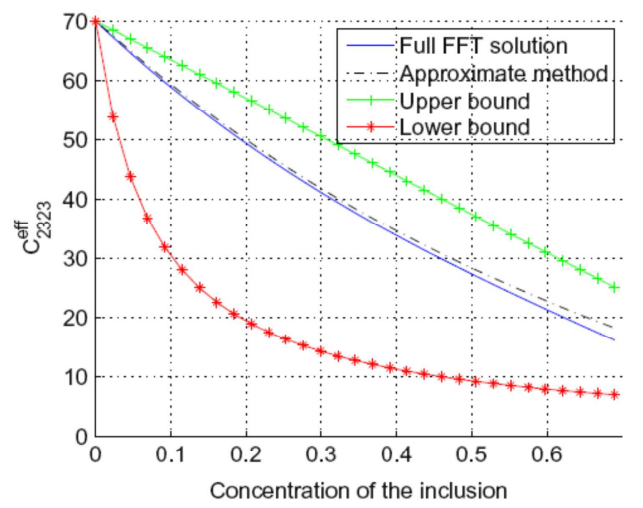

Fig. 6. Effective shear modulus obtained from the approximate solution compared with the solution obtained by the FFT method. Both are compared with the bounds of Voigt and Reuss.

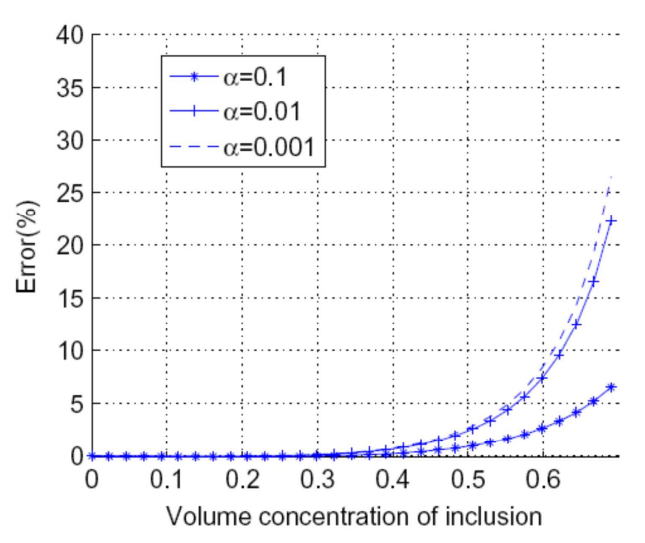

Fig. 7. Error on the approximate solution compared with the full FFT solution for different contrasts of the shear modulus.

\section{The viscoelastic solution}

Let us consider now a microstructure characterized by a cell having a cubic symmetry, the constituent materials being characterized by a matrix having a viscoelastic behavior of Kelvin, Maxwell or Burgers type and inclusions being linearly elastic. Using Laplace transform, the effective elasticity tensor in the Laplace-Carson space is given by (47):

$\mathbb{C}^{e f f}(s)=\mathbb{C}^{M}(s)-f \mathbb{D}(s)^{-1}$

or in the matrix form:

$$
\left[\mathbf{C}^{\text {eff }}(s)\right]=[\mathbf{C}(s)]-f \sum_{k=1}^{6} r_{k}(s)
$$

with:

$r_{k}(s)=\frac{1}{a_{k}(s)-\frac{u_{k}}{\lambda_{M}(s)+2 \mu_{M}(s)}-\frac{v_{k}}{2 \mu_{M}(s)}}$

The eigenvalues of the matrix $[\mathbf{U}]$ and $[\mathbf{V}]$ are given by:

$\{u\}=\left[\begin{array}{c}S_{4}+2 S_{7} \\ S_{4}-S_{7} \\ S_{4}-S_{7} \\ 2 S_{7} \\ 2 S_{7} \\ 2 S_{7}\end{array}\right]$

and:

$\{v\}=\left[\begin{array}{c}2 S_{1}-2 S_{4}-4 S_{7} \\ 2 S_{1}-2 S_{4}+2 S_{7} \\ 2 S_{1}-2 S_{4}+2 S_{7} \\ 2 S_{4}-4 S_{7} \\ 2 S_{4}-4 S_{7} \\ 2 S_{4}-4 S_{7}\end{array}\right]$

The non null components of matrix $[\mathbf{D}]^{-1}$ are given by:

$$
\begin{aligned}
& D_{11}=D_{22}=D_{33}=\frac{1}{3}\left(r_{1}(s)+2 r_{2}(s)\right) \\
& D_{12}=D_{13}=D_{23}=\frac{1}{3}\left(r_{1}(s)-r_{2}(s)\right) \\
& D_{44}=D_{55}=D_{66}=r_{4}(s)
\end{aligned}
$$


The components of the effective elasticity tensor in the Laplace-Carson space $\mathbf{C}^{\text {eff }}(s)$ are given in the following forms:

$C_{1111}^{\text {eff }}(s)=\frac{3 \kappa(s)+4 \mu(s)-f\left(r_{1}(s)+2 r_{2}(s)\right)}{3}$

$C_{1122}^{\text {eff }}(s)=\frac{3 \kappa(s)-2 \mu(s)-f\left(r_{1}(s)-r_{2}(s)\right)}{3}$

$C_{2323}^{\text {eff }}(s)=\frac{2 \mu(s)-\mathrm{fr}_{4}(s)}{2}$

In the following, we focus on isotropic materials for which the behavior of the matrix is linearly viscoelastic, according to models of Kelvin, Maxwell or Burgers, while inclusions are linear elastic. So the incompressibility and shear moduli in Laplace-Carson space are given by:

$$
\left\{\begin{array}{l}
\mu(s)=\frac{\mu_{N}(s)}{\mu_{D}(s)} \\
\kappa(s)=\frac{\kappa_{N}(s)}{\kappa_{D}(s)}
\end{array}\right.
$$

where $\mu_{N}, \mu_{D}, \kappa_{N}, \kappa_{D}$ are polynomials in $s$. Finally, the values of functions $r_{k}(s)$ are given by:

$r_{1}=\frac{6\left(3 G_{16}+4 G_{52}-\kappa_{l}\left(3 G_{56}+4 G_{32}\right)\right)}{8 G_{32}-9 v_{1}\left(G_{14}-\kappa_{l} G_{54}\right)+\omega_{1} G_{56}+\omega_{2} G_{36}}$

$r_{2}=\frac{2 \mu_{l}\left(3 G_{47}+4 G_{28}\right)-2\left(3 G_{210}+4 G_{29}\right)}{\phi_{1} G_{28}+3\left(v_{2}-1\right) G_{74}-\phi_{2} G_{94}-3 \mu_{1} v_{2} G_{104}}$

$r_{4}=\frac{2 \mu_{I}\left(3 G_{47}+4 G_{28}\right)-2\left(3 G_{210}+4 G_{29}\right)}{\chi_{1} G_{28}+3\left(v_{4}-1\right) G_{74}-\chi_{2} G_{94}-3 \mu_{1} v_{4} G_{104}}$

where:

$$
\begin{array}{r}
\omega_{1}=\left(6-18 u_{1}-12 v_{1}\right) \\
\omega_{2}=\kappa_{I}\left(18 u_{1}+12 v_{1}\right) \\
\phi_{1}=6 u_{2}+4 v_{2}-4 \\
\phi_{2}=\mu_{l}\left(6 u_{2}+4 v_{2}\right) \\
\chi_{1}=6 u_{4}+4 v_{4}-4 \\
\chi_{2}=\mu_{l}\left(6 u_{4}+4 v_{4}\right) \\
G_{i j}=F_{i} F_{j} \quad(i, j=1 . .10)
\end{array}
$$

where functions $F_{i}$ are given by:

$$
\begin{array}{rr}
F_{1}=\kappa_{N}^{2}(s) & F_{2}=\mu_{N}^{2}(s) \\
F_{3}=\kappa_{D}^{2}(s) & F_{4}=\mu_{D}^{2}(s) \\
F_{5}=\kappa_{N}(s) \kappa_{D}(s) & F_{6}=\mu_{N}(s) \mu_{D}(s) \\
F_{7}=\kappa_{N}(s) \mu_{N}(s) & F_{8}=\kappa_{D}(s) \mu_{D}(s) \\
F_{9}=\kappa_{D}(s) \mu_{N}(s) & F_{10}=\kappa_{N}(s) \mu_{D}(s)
\end{array}
$$

This set of equations shows that the effective moduli are all rational fractions of the Laplace variable s. Its inverse can be obtained explicitly as shown before in Section 2 .

\section{Numerical applications in the viscoelastic case}

6.1. Examples of 3D composites with inclusions on a simple cubic lattice

To illustrate the method described above, an application is made in the case of a microstructure with inclusions distributed over a simple cubic lattice. As previously, the inclusions are assumed elastic and the matrix is viscoelastic of isotropic Kelvin-Voigt type. From Appendix D3, the relaxation functions in Laplace-Carson space are given by:

$$
\begin{aligned}
& R_{\kappa}^{*}=\kappa+\eta_{\kappa} s \\
& R_{\mu}^{*}=\mu+\eta_{\mu} S
\end{aligned}
$$

The constituent materials are isotropic and the structure is cubic. So, the effective tensor possesses the cubic geometry, so that the elasticity fourth-rank tensor $\mathbb{R}(t)$ depends only on three independent functions, namely:

- An effective relaxation function in compression $\kappa_{\text {eff }}(t)$ :

$$
\kappa_{\text {eff }}(t)=\frac{C_{1111}^{\text {eff }}(t)+2 C_{1122}^{\text {eff }}(t)}{3}
$$

- Two effective relaxation functions in shear $\mu_{\text {eff }}(t)$ et $\mu_{\text {eff }}^{*}(t)$ :

$$
\begin{aligned}
& \mu_{\text {eff }}(t)=C_{2323}^{\text {eff }}(t) \\
& \mu_{\text {eff }}^{*}(t)=\frac{C_{1111}^{\text {eff }}(t)-C_{1122}^{\text {eff }}(t)}{2}
\end{aligned}
$$

A numerical application is presented with the parameters defined in Table 2.

Figs. 8-10 show the effective relaxation functions versus time for different wave-number discretizations used in the computation of terms $S_{k}$. The results show the effect of wave-number discretization on the relaxation functions appears to be less important than in the case of elastic constants. Indeed, the viscoelastic computations synthetize numerous "pseudo-elastic" materials at different values of $s$ and the synthetized results seem to be less sensitive to wave-number discretization.

The results being obtained in the form of a rational fraction, it was possible to recover a rheological model for each partial relaxation function of the effective behavior. The rheological model is the same for all three partial relaxation functions and given in Fig. 11, the physical properties of rheological components being described in Table 3.

Table 2

Mechanical properties of a periodic composite containing a viscoelastic matrix of Kelvin-Voigt type.

\begin{tabular}{lcc}
\hline Phase & Inclusion & Matrix \\
\hline Volume concentration (\%) & 15 & 85 \\
Incomp. modulus $(\mathrm{GPa})$ & 70 & 20 \\
Shear modulus $(\mathrm{GPa})$ & 30 & 5 \\
Viscosity in comp. $(\mathrm{GPa} \cdot$ day $)$ & 0 & 7 \\
Viscosity in shear $(\mathrm{GPa} \cdot$ day) & 0 & 5 \\
\hline
\end{tabular}




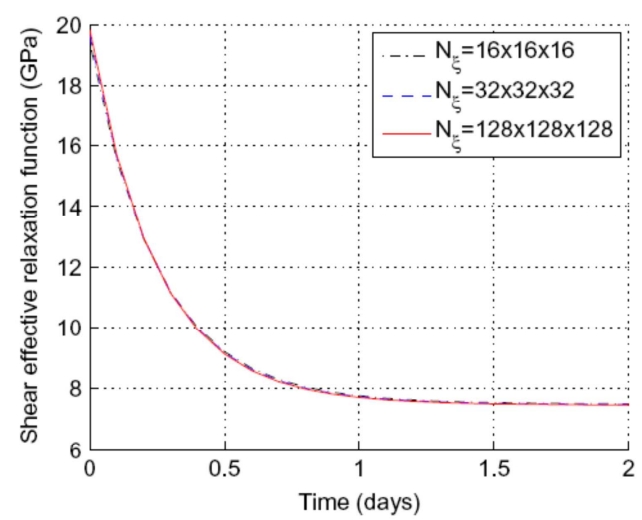

Fig. 8. Relaxation function for the first effective shear modulus of a composite with a viscoelastic matrix of Kelvin-Voigt type obtained from the FFT approximate solution for different wave-number discretizations.

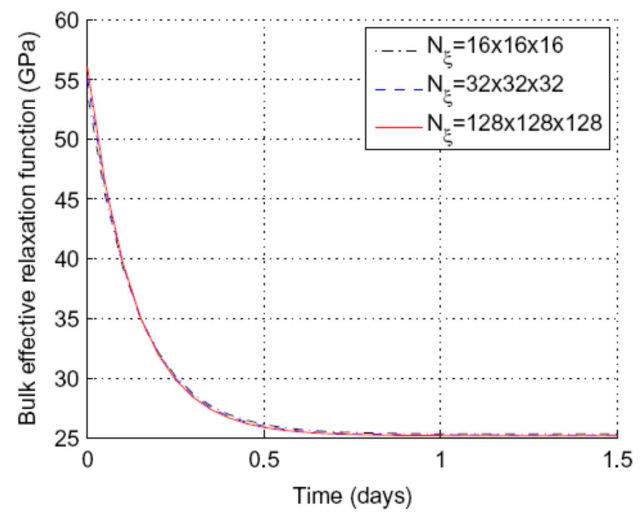

Fig. 9. Relaxation function for the effective bulk modulus of a composite with a viscoelastic matrix of Kelvin-Voigt type obtained from the FFT approximate solution for different wave-number discretizations.

To demonstrate the flexibility of the method, the case of rheological models of Maxwell and Burgers types were also studied. The results corresponding to these other rheological models are reported in A.1 and A.2.

\section{Validation of the method on a result of the literature}

In this section, the results coming from the approximate FFT solution are compared with those coming from an time iterative solution used by Lahellec and Suquet (2007). The material under study is a fiber reinforced composite consisting of two phases. The fiber axis is aligned along $x_{3}$ axis and the material of the fibers will be identified as phase 1 . This phase is elastic, linear, isotropic and characterized by the bulk modulus $\kappa^{(1)}=222.2 \mathrm{GPa}$ and the shear modulus $2 \mu^{(1)}=333.3 \mathrm{GPa}$. Its volume concentration is given by $c_{1}=0.25$. The behavior of the matrix is viscoelastic of Maxwell type, characterized by the incompressibility coefficient $k^{(2)}=58.33 \mathrm{GPa}$, shear $2 \mu^{(2)}=53.84 \mathrm{GPa}$ and viscosity $\eta^{(2)}=10 \mathrm{GPa}$.

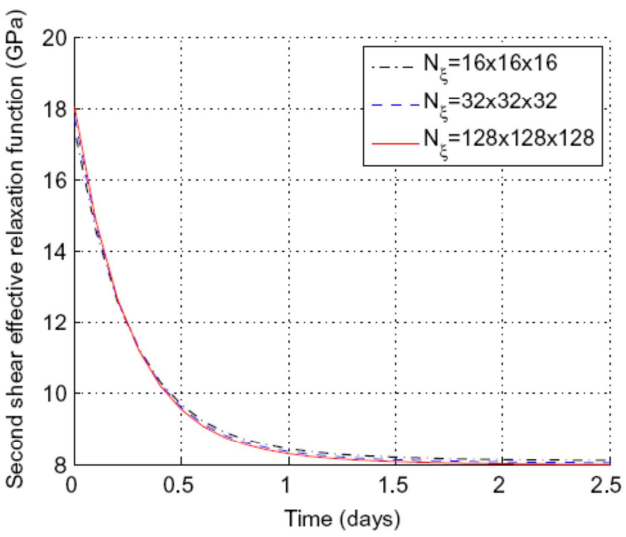

Fig. 10. Relaxation function for the second effective shear modulus of a composite with a viscoelastic matrix of Kelvin-Voigt type obtained from the FFT approximate solution for different wave-number discretizations.

The problem is two-dimensional and the loading is characterized by giving a time path description of the macroscopic strain as:

$\mathbb{E}(t)=E_{11}(t) \mathbf{e}_{1} \otimes \mathbf{e}_{1}-E_{11}(t) \mathbf{e}_{2} \otimes \mathbf{e}_{2}$

where: $E_{11}(t)$ is a monotonically increasing function with a constant strain rate $\dot{E}_{11}=5.10^{-3} \mathrm{~s}^{-1}$

Fig. 12 shows the variation of the macroscopic stress $\Sigma_{11}$ as a function of time obtained by the approximate FFT solution and by Lahellec and Suquet (2007). The result shows that both results are identical, which validates again the results coming from the approximate FFT solution used in the present work.

\section{Conclusion}

In this paper, we proceeded to the determination of viscoelastic properties of periodic inclusion-matrix composites by an approximate FFT solution based on a simplification of the solution of the integral equation for heterogeneous media. The effect of the microstructure is accounted for by using explicitly the distribution of inclusions through the characteristic function of the domain containing the inclusions. The overall modulus in Laplace-Carson's space for this type of solution presents itself as a rational fraction, which allows the semi-analytical inversion of the Laplace transform. This procedure allows to calculate explicitly the relaxation function of the material. The examples tested in the case of periodic microstructures of 3D simple cubic array of spheres or 2D array of fibers have shown that the proposed method is able to deal with the case of different types of behavior for the matrix. A validation was performed successfully by comparison with results coming from time-step integration of the solution. It is worthwhile mentioning that the method rests on shape functions which are the Fourier components of the characteristic function of the domain containing the inclusions. As such, it allows to account for complex distributions coming for example from 3D tomography of the microstructure. 


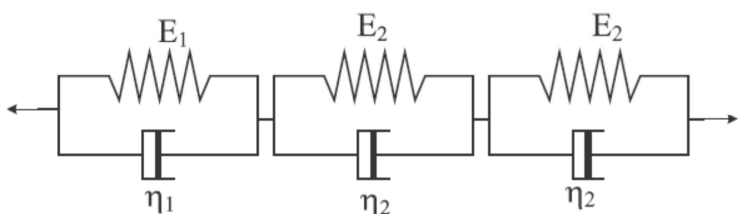

Fig. 11. Rheological model associated with the macroscopic behavior of a viscoelastic periodic composite comprising a matrix of Kelvin-Voigt type using the FFT approximation.

Table 3

Viscoelastic properties of rheological model associated with the macroscopic behavior of a periodic composite containing a matrix of Kelvin-Voigt type as obtained by the FFT approximation.

\begin{tabular}{lrrr}
\hline Properties & Element 1 & Element 2 & Element 3 \\
\hline Modulus & $(G P a)$ & & \\
Shear & 46.57 & 187.77 & 9.32 \\
Buld & 133.23 & 32.44 & 1.86 \\
Second shear & 44.68 & 38.07 & 13.04 \\
Viscocity & $($ GPa $\cdot$ day $)$ & & \\
Shear & 4.97 & 100.35 & 9.32 \\
Bulk & 6.11 & 13.15 & 1.87 \\
Second shear & 5.38 & 22.73 & 13.04 \\
\hline
\end{tabular}

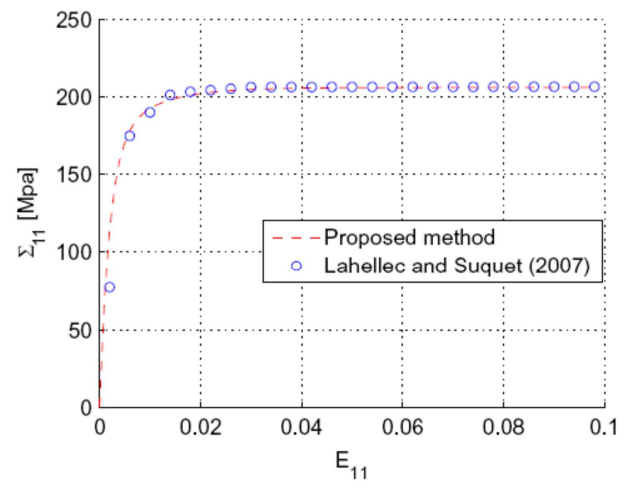

Fig. 12. Response of a fiber reinforced composites under monotonic loading. Comparison between the results of the FFT method and those obtained by (Lahellec and Suquet, 2007).

Appendix A. Applications of the approximate FFT solution for different rheological models

\section{A.1. Maxwell's model}

A numerical application is presented for a rheological model of Maxwell type with the parameters defined in Table A.4 defining the different parts of the model. The relaxation function for the overall bulk modulus is reported in Fig. A.13 while the ones related to both overall shear moduli are reported in Figs. A.14 and A.15. These results show that the convergence is still very good and ensured for 64 wavenumbers along each direction.
Table A.4

Mechanical properties of a periodic composite containing a viscoelastic matrix of Maxwell type.

\begin{tabular}{lcc}
\hline Phase & Inclusion & Matrix \\
\hline Volume concentration $(\%)$ & 50 & 50 \\
Incomp. modulus $(\mathrm{GPa})$ & 100 & 60 \\
Shear modulus $(\mathrm{GPa})$ & 30 & 45 \\
Viscosity in comp. $(\mathrm{GPa} \cdot$ day $)$ & 0 & 14 \\
Viscosity in shear $(\mathrm{GPa} \cdot$ day $)$ & 0 & 5 \\
\hline
\end{tabular}

\section{A.2. Burgers's model}

A numerical application is now presented for a Bur gers's model which comprises two different parts: one being built as a Maxwell model while the other is built as a Kelvin model. The set of all the necessary parameters is defined in Table A.5. Despite the larger set of rheological parameters, the method can still be applied with success as shown by Figs. A.16, A.17 and A.18 displaying the relaxation functions for the bulk modulus and shear moduli. This shows again the ability of the method to deal with various rheological models for the constituents of the composite material.

\section{Appendix B. Decomposition in the Walpole's base}

We recall the definition of the Walpole's base (Walpole, 1981) for tensors of order four with a transverse isotropic symmetry. This base has some interesting properties for performing the product between such tensors. From a general point of view, transverse isotropy is characterized by a plane within which isotropy is present. The direction of the plane is characterized by the direction of the normal to the plane. In the case of wave-number decompositon, the unit normal to the plane is given for each wave vector by the unit vector along the direction of the wave vector. Finally, the elements of the Walpole's base are defined by:

$$
\left\{\begin{array}{l}
\mathbb{E}_{1}(\xi)=\frac{1}{2} \mathbf{k}^{\perp} \otimes \mathbf{k}^{\perp} \\
\mathbb{E}_{2}(\xi)=\mathbf{k} \otimes \mathbf{k} \\
\mathbb{E}_{3}(\xi)=\mathbf{k}^{\perp} \otimes \mathbf{k}^{\perp}-\mathbb{E}_{1} \\
\mathbb{E}_{4}(\xi)=\mathbf{k}^{\perp} \otimes \mathbf{k}+\mathbf{k} \otimes \mathbf{k}^{\perp} \\
\mathbb{E}_{5}(\xi)=\mathbf{k} \otimes \mathbf{k}^{\perp}, \quad \mathbb{E}_{6}(\xi)=\mathbf{k}^{\perp} \otimes \mathbf{k}
\end{array}\right.
$$

where $\mathbf{k}$ et $\mathbf{k}^{\perp}$ is defined by:

$$
\mathbf{k}=\frac{1}{|\xi|^{2}} \xi \otimes \xi, \quad \mathbf{k}^{\perp}=\mathbf{i}-\mathbf{k}
$$




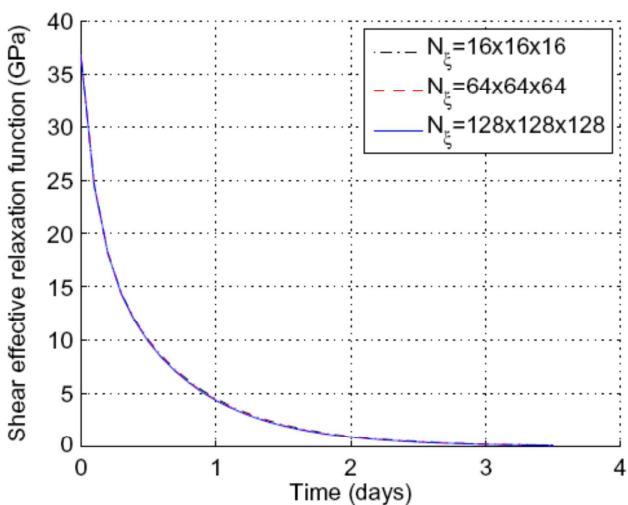

Fig. A.13. Relaxation function for the effective bulk modulus of a composite with a viscoelastic matrix of Maxwell type.

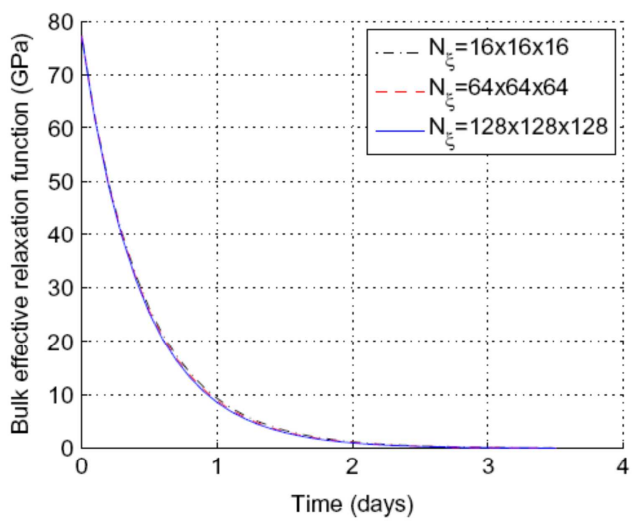

Fig. A.14. Relaxation function for the first effective shear modulus of a composite with a viscoelastic matrix of Maxwell type.

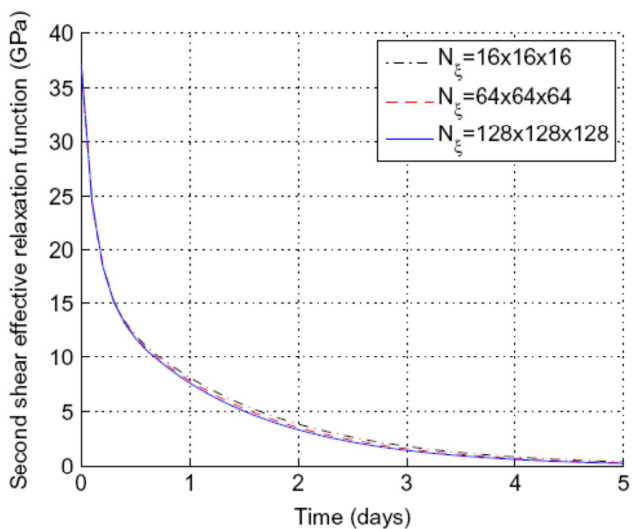

Fig. A.15. Relaxation function for the second effective shear modulus of a composite with a viscoelastic matrix of Maxwell type.
Table A.5

Mechanical properties of a periodic composite containing a viscoelastic matrix of Burgers type.

\begin{tabular}{lcll}
\hline Phase & Inc. & Matrix \\
\hline & & Max. & Kel. \\
\hline Volume concentration (\%) & 30 & 70 & \\
Incomp. modulus $(\mathrm{GPa})$ & 70 & 60 & 40 \\
Shear modulus $(\mathrm{GPa})$ & 20 & 30 & 25 \\
Bulk viscocity $(\mathrm{GPa} \cdot$ day) & 0 & 5 & 35 \\
Shear viscocity $(\mathrm{GPa} \cdot$ day $)$ & 0 & 2 & 12 \\
\hline
\end{tabular}

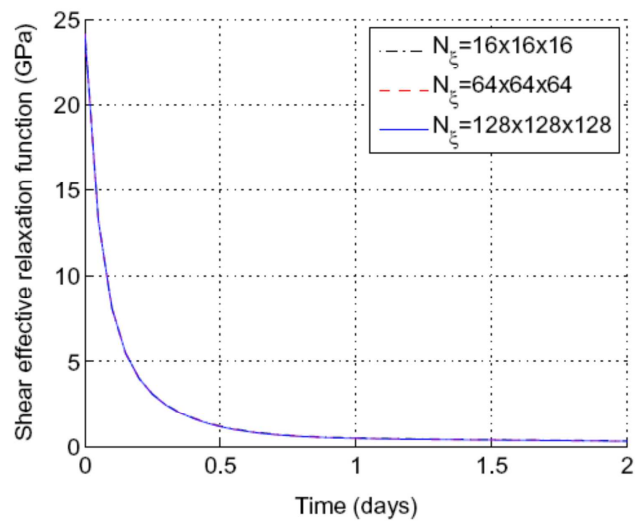

Fig. A.16. Relaxation function for the effective bulk modulus of composite with a viscoelastic matrix of Burgers type.

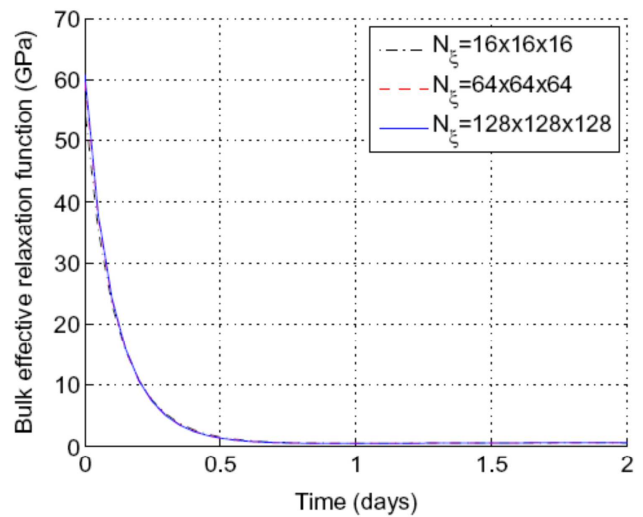

Fig. A.17. Relaxation function for the effective shear modulus of a composite with a viscoelastic matrix of Burgers type.

with the condition $|\xi| \neq 0$.

For an isotropic material, the Green's tensor $\boldsymbol{\Gamma}$ is given by:

$\Gamma=\frac{1}{\lambda+2 \mu} \mathbb{E}_{2}+\frac{1}{2 \mu} \mathbb{E}_{4}$

\section{Appendix C. Components of tensors $\mathbb{G}^{(1)}$}

Full expressions of tensors $\mathbb{G}^{(1)}$ are given by: 


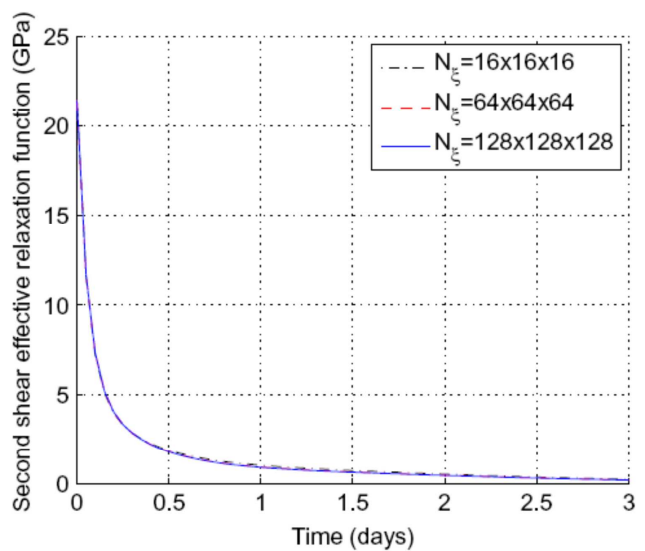

Fig. A.18. Relaxation function for the second effective shear modulus of a composite with a viscoelastic matrix of Burgers type.

$$
\left\{\begin{array}{l}
G_{i j k l}^{(1)}=\frac{1}{2}\left\{\bar{\xi}_{j}\left(\delta_{i l} \bar{\xi}_{k}+\delta_{i k} \bar{\xi}_{l}\right)+\bar{\xi}_{i}\left(\delta_{j l} \bar{\xi}_{k}+\delta_{j k} \bar{\xi}_{l}\right)\right\} \\
G_{i j k l}^{(2)}=\bar{\xi}_{i} \bar{\xi}_{j} \bar{\xi}_{k} \bar{\xi}_{l}
\end{array}\right.
$$

where $\left(\bar{\xi}_{s}\right)$, is the unit vector along the direction of the wave vector $\xi_{s}$. For a parallelepipedic cell with three orthogonal planes of symmetry, only 9 terms are involved:

$$
\left\{\begin{array}{lll}
h_{1}=\left(\bar{\xi}_{1}\right)^{2}, & h_{2}=\left(\bar{\xi}_{2}\right)^{2}, & h_{3}=\left(\bar{\xi}_{3}\right)^{2} \\
h_{4}=\left(\bar{\xi}_{1}\right)^{4}, & h_{5}=\left(\bar{\xi}_{2}\right)^{4}, & h_{6}=\left(\bar{\xi}_{3}\right)^{4} \\
h_{7}=\left(\bar{\xi}_{2} \bar{\xi}_{3}\right)^{2}, & h_{8}=\left(\bar{\xi}_{3} \bar{\xi}_{1}\right)^{2}, & h_{9}=\left(\bar{\xi}_{1} \bar{\xi}_{2}\right)^{2}
\end{array}\right.
$$

With the previous notation, the matrices associated with tensors $\mathbb{G}$ are given by:

$$
\begin{aligned}
& {\left[G^{(1)}\right]=\left[\begin{array}{cccccc}
2 h_{1} & 0 & 0 & 0 & 0 & 0 \\
0 & 2 h_{2} & 0 & 0 & 0 & 0 \\
0 & 0 & 2 h_{3} & 0 & 0 & 0 \\
0 & 0 & 0 & h_{2}+h_{3} & 0 & 0 \\
0 & 0 & 0 & 0 & h_{3}+h_{1} & 0 \\
0 & 0 & 0 & 0 & 0 & h_{1}+h_{2}
\end{array}\right]} \\
& {\left[G^{(2)}\right]=\left[\begin{array}{cccccc}
h_{4} & h_{9} & h_{8} & 0 & 0 & 0 \\
h_{9} & h_{5} & h_{7} & 0 & 0 & 0 \\
h_{8} & h_{7} & h_{6} & 0 & 0 & 0 \\
0 & 0 & 0 & 2 h_{7} & 0 & 0 \\
0 & 0 & 0 & 0 & 2 h_{8} & 0 \\
0 & 0 & 0 & 0 & 0 & 2 h_{9}
\end{array}\right]}
\end{aligned}
$$

\section{Appendix D. Tensorial decomposition of isotropic elasticity and viscoelasticity tensors}

\section{D.1. Elasticity behavior}

In the case of a heterogeneous material where each phase has an isotropic elastic behavior, defined by the shear modulus $\mu$ and incompressibility $\kappa$, the stiffness tensor $\mathbb{C}$ and the compliance tensor $\mathbb{S}$ can be expressed as Zaoui, 1998:
$\left\{\begin{array}{l}\mathbb{C}=2 \mu \mathbb{K}+3 \kappa \rrbracket \\ \mathbb{S}=\frac{1}{2 \mu} \mathbb{K}+\frac{1}{3 \kappa} \rrbracket\end{array}\right.$

where fourth-order tensors $\mathbb{k}$ and $\Downarrow$ are spherical and deviatoric parts, respectively, and defined by:

$$
\left\{\begin{array}{l}
\mathbb{J}=\frac{1}{3} \mathbf{i} \otimes \mathbf{i} \\
\mathbb{K}=\mathbb{\square}-\mathbb{J}
\end{array}\right.
$$

where $\mathbf{i}$ is the second order identity tensor. The interest in using fourth-order tensors $\mathbb{K}$ and $\Downarrow$ comes from the following relations:

$$
\left\{\begin{array}{lll}
\mathbb{U}: \mathbb{}=\mathbb{V} & \mathbb{K}: \mathbb{K}=\mathbb{K} & \mathbb{J}: \mathbb{J}=\mathbb{} \\
\mathbb{K}: \mathbb{V}=0 & \mathbb{Q}: \mathbf{i}=\mathbb{J}: \mathbf{i}=\mathbf{i} & \mathbb{K}: \mathbf{i}=0 \\
\mathbb{J}: \mathbb{K}=0 & \mathbb{K}: \boldsymbol{\epsilon}=\mathbf{e} & \mathbb{J}: \boldsymbol{\epsilon}=\frac{1}{3} \operatorname{tr}(\boldsymbol{\epsilon}) \mathbf{i}
\end{array}\right.
$$

where $\mathbf{e}$ is the deviator of a second order symmetric tensor $\boldsymbol{\epsilon}$.

\section{D.2. Viscoelastic behavior of isotropic Kelvin-Voigt's type}

The isotropic viscoelastic behavior of the Kelvin-Voigt's type is given by:

$\boldsymbol{\sigma}=\mathbb{C}: \boldsymbol{\epsilon}+\boldsymbol{\eta}: \dot{\boldsymbol{\epsilon}}$

where $\boldsymbol{\eta}$ is the fourth-order tensor, defined by:

$\boldsymbol{\eta}=3 \eta_{\kappa} \rrbracket+2 \eta_{\mu} \mathbb{K}$

This produces in the Laplace-Carson space:

$\boldsymbol{\sigma}^{*}=(\mathbb{C}+\boldsymbol{\eta} s): \boldsymbol{\epsilon}^{*}$

which gives:

$\boldsymbol{\sigma}^{*}=\left[3\left(\kappa+\eta_{\kappa} s\right) \rrbracket+2\left(\mu+\eta_{\mu} s\right) \mathbb{K}\right]: \boldsymbol{\epsilon}^{*}$

or:

$\sigma^{*}=\mathbb{R}^{*}: \epsilon^{*}$

with:

$\mathbb{R}^{*}=3 R_{\kappa}^{*} \mathbb{d}+2 R_{\mu}^{*} \mathbb{K}$

and:

$\left\{\begin{array}{l}R_{\kappa}^{*}=\kappa+\eta_{\kappa} s \\ R_{\mu}^{*}=\mu+\eta_{\mu} s\end{array}\right.$

where $\eta_{\kappa}, \eta_{\mu}$ are the viscosity in shear and in compression, respectively.

D.3. Viscoelastic behavior of the isotropic Maxwell's type

The isotropic viscoelastic behavior of Maxwell type is given by:

$\dot{\boldsymbol{\epsilon}}=\mathbb{C}^{-1}: \dot{\boldsymbol{\sigma}}+\boldsymbol{\eta}^{-1}: \boldsymbol{\sigma}$

This produces in the Laplace-Carson's space:

$\boldsymbol{\epsilon}^{*}=\left(\mathbb{C}^{-1}+\frac{1}{s} \boldsymbol{\eta}^{-1}\right): \boldsymbol{\sigma}$

which gives: 
$\boldsymbol{\epsilon}^{*}=\left[\left(\frac{1}{3 \kappa}+\frac{1}{3 \eta_{\kappa} s}\right) \sqrt[J]{ }+\left(\frac{1}{2 \mu}+\frac{1}{2 \eta_{\mu} s}\right) \mathbb{K}\right]: \boldsymbol{\sigma}^{*}$

or:

$\boldsymbol{\sigma}^{*}=\mathbb{R}^{*}: \epsilon^{*}$

with:

$\left\{\begin{array}{l}R_{\kappa}^{*}=\frac{\kappa \eta_{K} s}{\kappa+\eta_{\kappa} s} \\ R_{\mu}^{*}=\frac{\mu \eta_{\mu} s}{\mu+\eta_{\mu} s}\end{array}\right.$

\section{References}

Iwakuma, T., Nemat-Nasser, S., 1983. Composites with periodic microstructure. Computers \& Structures $16(1-4), 13-19$.

Lahellec, N., Suquet, P., 2007. Effective behavior of linear viscoelastic composites: a time-integration approach. International Journal of Solids and Structures 44 (2), 507-529.

Nemat-Nasser, S., Iwakuma, T., Hejazi, M., 1982. On composites with periodic structure. Mechanics of Materials 1 (3), 239-267.

Nemat-Nasser, S., Yu, N., Hori, M., 1993. Bounds and estimates of overall moduli of composites with periodic microstructure. Mechanics of Materials 15 (3), 163-181.
Nemat-Nasser, S. Hori, M., 1993. Micromechanics: Overall Properties of Heterogeneous Materials, North-Holland.

Michel, J., Moulinec, H., Suquet, P.. 1999. Effective properties of composite materials with periodic microstructure: a computational approach. Computer Methods in Applied Mechanics and Engineering $172(1-4)$ 109-143.

Bonnet, G., 2007. Effective properties of elastic periodic composite media with fibers. Journal of the Mechanics and Physics of Solids 55 (5), 881-899.

Zeman, J., Vondrejc, J., Novk, J., Marek, I., 2010. Accelerating a FFT-based solver for numerical homogenization of periodic media by conjugate gradients. Journal of Computational Physics 229 (21), 8065-8071.

Moulinec, H., Suquet, P. 2003. Comparison of FFT-based methods for computing the response of composites with highly contrasted mechanical properties. Physica B: Condensed Matter 338 (1-4), 5860. Proceedings of the Sixth International Conference on Electrical Transport and Optical Properties of Inhomogeneous Media

Christensen, R.M., Freund, L.B., 1984. Theory of viscoelasticity, an introduction. Journal of Applied Mechanics 51, 226.

Salençon, J., 2009. Viscoélasticité pour le calcul des structures. Editions Ecole Polytechnique.

Walpole, L., 1981. Elastic behavior of composite materials: theoretica foundations. Advances in Applied Mechanics 21, 169-242.

Walpole, L., 1966. On bounds for the overall elastic moduli of inhomogeneous systems - I. Journal of the Mechanics and Physics of Solids 14 (3), 151-162.

Zaoui, A., 1998. Matériaux hétérogènes et composites. Editions Ecole Polytechnique. 